\title{
Fluxes of energetic protons and electrons measured on board the Oersted satellite
}

\author{
J. Cabrera ${ }^{1}$, M. Cyamukungu ${ }^{1}$, P. Stauning ${ }^{3}$, A. Leonov ${ }^{1,2}$, P. Leleux ${ }^{1}$, J. Lemaire ${ }^{1}$, and G. Grégoire ${ }^{1}$ \\ ${ }^{1}$ Center for Space Radiations (CSR), Chemin du cyclotron 2, 1348 Louvain-la-Neuve, Belgium \\ ${ }^{2}$ Moscow Engineering and Physics Institute (MEPHI), Kashirskoe Shosse 31, 115409 Moscow, Russia \\ ${ }^{3}$ Danish Meteorological Institute (DMI), Lyngbyveij 100, DK-2100, Copenhagen, Denmark
}

Received: 8 February 2005 - Revised: 25 April 2005 - Accepted: 6 May 2005 - Published: 22 November 2005

Part of Special Issue "1st European Space Weather Week (ESWW)"

\begin{abstract}
The Charged Particle Detector (CPD) on board the Oersted satellite $(649 \mathrm{~km}$ perigee, $865 \mathrm{~km}$ apogee and $96.48^{\circ}$ inclination) currently measures energetic protons and electrons. The measured peak fluxes of $\mathrm{E}>1 \mathrm{MeV}$ electrons are found to confirm the predictions of AE8-MAX, though they occur at a geographical position relatively shifted in the SAA. The fluxes of protons are one order of magnitude higher than the predictions of AP8-MAX in the energy range 20-500 MeV. This huge discrepancy between AP8 and recent measurements in LEO was already noticed and modelled in SAMPEX/PSB97 and TPM-1 models. Nevertheless some other LEO measurements such as PROBA and CORONA$F$ result in flux values in good agreement with AP8 within a factor 2. The anisotropy of the low-altitude proton flux, combined with measurement performed on board three-axis stabilised satellites, has been suspected to be one possible source of the important discrepancies observed by different missions. In this paper, we evaluate the effect of anisotropy on flux measurements conducted using the CPD instruments. On the basis of the available data, we confirm the inaccuracy of AP8 at LEO and suggest methods to improve the analysis of data in future flux measurements of energetic protons at low altitudes.
\end{abstract}

Keywords. Magnetospheric physics (Energetic particles, trapped) - Ionosphere (Ionosphere-magnetosphere interactions) - Space plasma physics (Instruments and techniques)

\section{Introduction}

The Oersted satellite was launched on 23 February 1999 on a LEO orbit (649 km perigee, $865 \mathrm{~km}$ apogee, $96.48^{\circ}$ inclination). Its main mission is to accurately measure the mag-

Correspondence to: J. Cabrera

(cabrera@spaceradiations.be) netic field along its orbit. This satellite is three-axis stabilised by magnetic torquers and a gravity gradient boom on which magnetometers are accommodated. The CPD detector on board the Oersted satellite has two of its six modules oriented towards the earth-to-satellite line and measure electron $(\mathrm{E}>1 \mathrm{MeV})$ and proton $(\mathrm{E}>20 \mathrm{MeV})$ fluxes mainly encountered in the South Atlantic Anomaly (SAA) and in the polar regions. The flux of energetic protons is known to be highly anisotropic (Lemaire et al., 1998) on the Oersted low altitude orbit. However, in order to achieve particle identification using the six channels of the instrument, the best constant approximate of the angle-dependant flux was searched for in the first phase of the CPD data analysis process. This resulted in electron and proton energy-dependent isotropic fluxes (Cyamukungu et al., 2001b). In a second phase, the approximation systematic error ensuing from the isotropy assumption was evaluated as a function of the parameter $n$ of the pitch angle distribution $F(\theta) \propto \sin ^{n} \theta$ and of the satellite orientation relatively to the local magnetic field.

A method to measure the angular and energy spectra of space radiations with simple sensors, while achieving particle identification has been described in (Cyamukungu et al., 2001a). For non-spinning satellite, the condition for this complete spectrum determination is that the intrinsic detection efficiencies of channels should be a non-constant function of particle energy, incidence angle and particle type.

More complex instruments like the NINA detector may perform measurements of angular distributions, energy and mass spectra although their handling of high counting rates or identification of very energetic particles may not be achieved (Leonov et al., 2005, Bidoli et al., 2001).

Instruments with an intermediate complexity devoted to the detection of one particle type may be used to measure pitch angle distribution and energy spectra using a method applied to analyse data from the German satellite Dial (Fischer et al., 1977). 

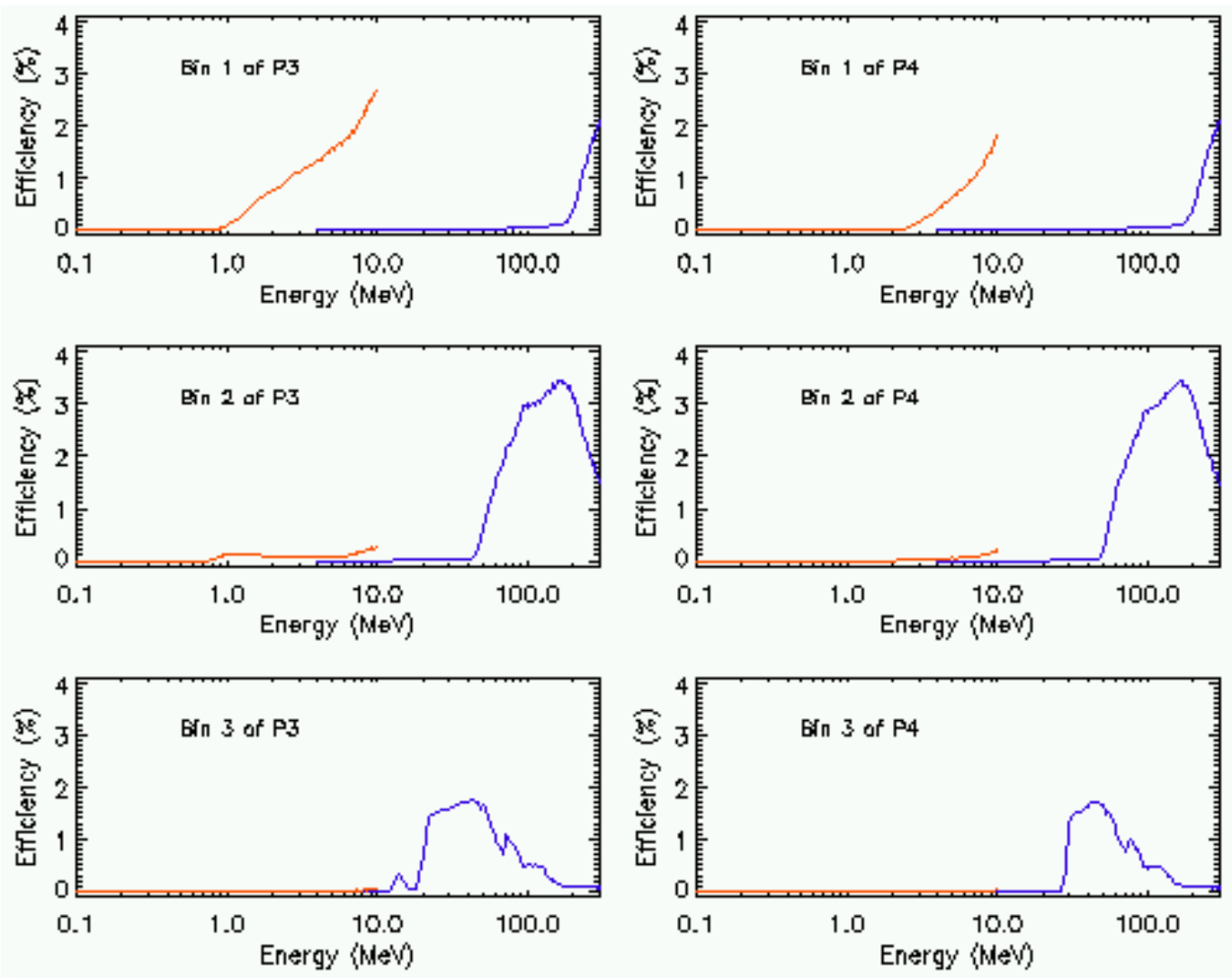

Fig. 1. Efficiencies of the CPD channels for electrons (red) and protons (blue) under the isotropic flux assumption.

The two-step method outlined above and applied to the CPD data is efficient whenever energy spectra and particle identification have to be derived from large field-of-view (FOV) detectors characterised by an almost constant efficiency over the FOV angle and lacking incidence angle measurement capability for every single particle. It was also applied in data analysis for instruments like the SREM, onboard PROBA (Buehler, 2003). Obviously the results from these instruments based on the isotropy assumption at a given space position may be different depending on their orientation especially on LEO altitude where the flux is highly anisotropic. Rough comparisons of the fluxes from some of these instruments and AP8 show an order of magnitude discrepancy. It led to question the validity of the comparison methods. This paper is devoted to the assessment of rules to apply while comparing fluxes derived under the isotropy assumption with omni-directional fluxes contained in models like AP8.

A brief description of the CPD is presented in Sect. 2 along with the methods applied in raw data analysis. Proton and electron flux results are presented in Sect. 3 and a critical analysis of the consequences of the isotropy assumption on the proton flux measurements at low altitude is given in Sect. 4.

\section{The CPD instrument}

The Charged Particle Detector (CPD) on board the Oersted satellite is an array of four upward-looking silicon sensors two of which are replicated and oriented toward the perpendicular to the boom direction (Cyamukungu et al., 2001b). Among the four up looking sensors, two are heavily shielded and devoted to the detection of energetic electrons and protons. These two energetic particle modules, hereafter named P3 and P4 are not replicated on the perpendicular direction. The energy range detected by each of these detectors has been divided into four energy intervals referred to as energy bins or energy channels. The bin number $\mathrm{j}$ of module $\mathrm{P}_{i}$ is also named $\mathrm{P}_{i j}$. In order to evaluate electron and proton contributions to particle fluxes measured by the CPD, counting rates of the channels $\mathrm{P} 31, \mathrm{P} 32$ and $\mathrm{P} 33$ of the module P3 were taken into account, in addition to channels P41, P42 and P43 selected from the module P4. Channels P34 and P44 were discarded. Simulations based on the GEANT4 software (Agostinelli et al., 2003) revealed that these channels contain information on proton and $\alpha$-particle fluxes but their in-flight counting rates are so low that they do not contribute significantly to the determination of the parameters of particle fluxes. The six CPD channels listed above were used to derive electron and proton energy spectra. Practically, for each particle type, two parameters of a power law spectrum 
were derived by a least-square fit of model counting rates to observed ones. The angular distribution was approximated by a constant (isotropic) flux over the $67^{\circ} \mathrm{FOV}$ angle. This constant flux is constrained by the fact that it would result in the same counting rates as those observed in $\mathrm{P} 3$ and $\mathrm{P} 4$ channels. For such a flux the geometrical factor of the detector aperture is $9 \mathrm{~cm}^{2} \mathrm{sr}$. The detection efficiencies of all the six CPD channels used to derive electron and proton spectra are shown in Fig. 1.

We recall that the intrinsic detection efficiency is the probability for a particle that crosses the detector aperture to be detected in a given channel. Therefore, efficiency functions fully characterize the detection capability of an instrument. In particular, the angle averaged efficiencies shown in Fig. 1 contain all the information on the energy range covered by each channel for electrons and protons. Accurate particle fluxes may be derived from counting rates of any channel shown in Fig. 1 using its efficiency. However, this is only possible if the corresponding silicon sensor is appropriately shielded and collimated. If particles are likely to reach the sensors trough any other path than the collimator cut-out, then the whole satellite must be included into simulation to evaluate global efficiency functions for the defined channels. The total mass of the $72 \times 45 \times 34 \mathrm{~cm}^{3}$ Oersted satellite is about $60 \mathrm{~kg}$ (50 kg dry mass). Most of this mass is at the back of the CPD even after the deployment of the boom initially stowed in the cylinder shown in Fig. 2a. The CPD module $\mathrm{P} 3$ and $\mathrm{P} 4$ are accommodated in the middle of the sensor row. The side and the aperture of these assemblies are mainly shielded by a brass collimator completed by a $1 \mathrm{~mm}$ thick copper (P4) or aluminium (P3) plate shown in Fig. 2b.

The brass collimator and the back side materials constitute efficient shieldings of silicon sensors inside P3 and P4 against energetic electrons (at least up to $7 \mathrm{MeV}$ ) from the satellite sides. Energetic protons (at least up to $100 \mathrm{MeV}$ ) from the back side are prevented from hitting the sensors. However, even though energetic protons from the front side are efficiently stopped by the brass collimators, the lack of mass on this side has offered paths to very energetic protons which have generated background counts in the CPD channels. A precise evaluation of this background would only be possible if the whole mass distribution of the satellite was included in the efficiency calculations. Without such a precise background evaluation, it was necessary to estimate its upper limit in a typical channel. Consider that protons from the front are allowed to hit the $\mathrm{P} 4$ sensor through the $1 \mathrm{~mm}$ thick copper shield, despite the collimator and any other shielding material., The efficiency curves show that they will be counted in channel P42, provided their incident energy is higher than $60 \mathrm{MeV}$. The geometrical factor of the silicon sensor exposed to a proton flux in a whole half-hemisphere is $\mathrm{G}=\pi \mathrm{A}=1.57 \mathrm{~cm}^{2} \mathrm{sr}$ (where $\mathrm{A}=50 \mathrm{~mm}^{2}$ is the sensor area). The maximum AP8-MAX flux of $\mathrm{E}>60 \mathrm{MeV}$ protons on Oersted orbit is $289 \mathrm{~cm}^{-2} \mathrm{sr}^{-1} \mathrm{~s}^{-1}$, which would result in a count rate of 453 protons/s. The observed maximum count rates in channel $\mathrm{P} 42$, for each month over the quiet time period from April to October 1999 are about 5 times higher
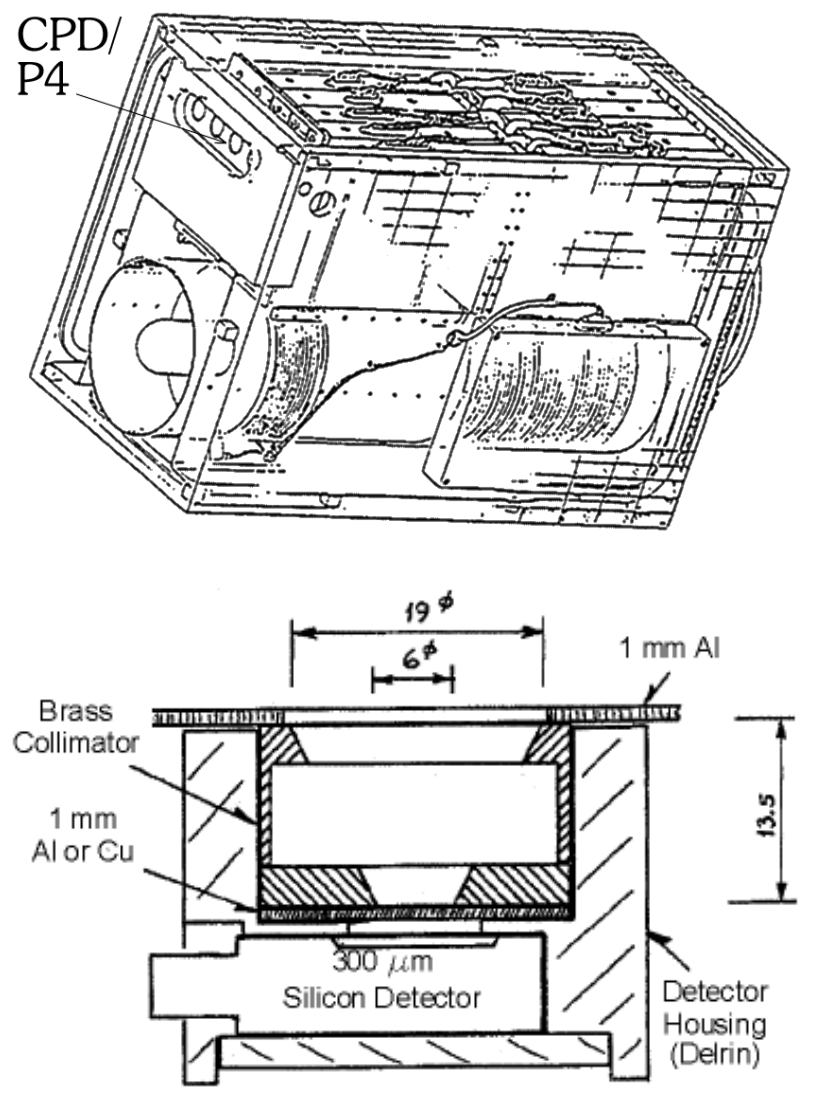

Fig. 2. (a) Oersted main body with stowed boom and the CPD, (b) P3 and P4 detector assembly.

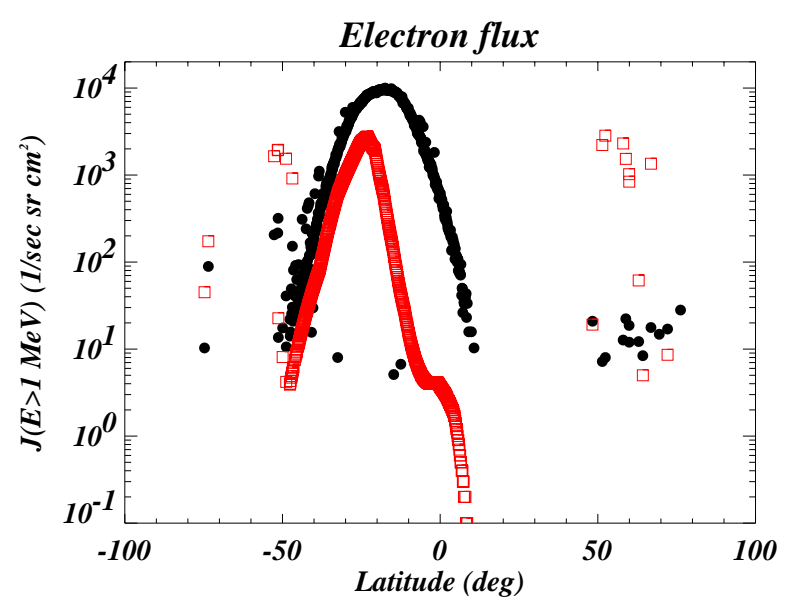

Fig. 3. Flux of energetic $(\mathrm{E}>1 \mathrm{MeV})$ electrons measured by the CPD (round) and AE8 model prediction (square) as a function of latitude on day 100 since 1st January 1999, at 14:52 UT. Typical statistical uncertainties on CPD electron fluxes amount to $30 \%$, but the systematic error is considered negligible.

than the above estimated AP8-MAX upper limit. This clearly demonstrates that the observed order of magnitude difference between AP8-MAX and Oersted/CPD fluxes cannot be mainly ascribed to background counts. 

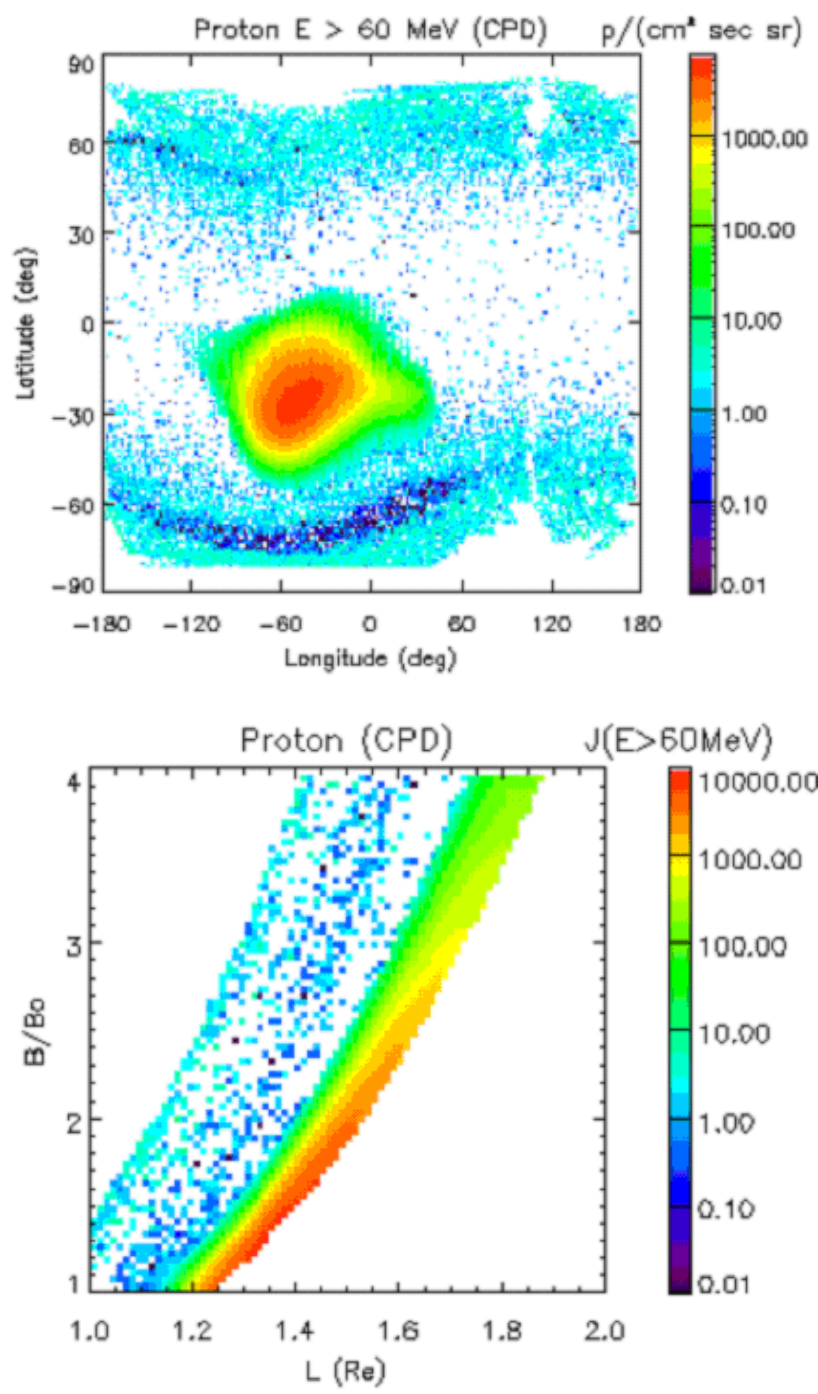

Fig. 4. Flux of energetic $(\mathrm{E}>60 \mathrm{MeV})$ protons measured by the CPD as a function of (a) the geographic (longitude, latitude) and (b) the $(\mathrm{B}, \mathrm{L})$ coordinates.

\section{Fluxes of energetic proton and electrons measured by the CPD}

The integral flux of high energy electrons $\mathrm{J}(\mathrm{E}>1 \mathrm{MeV})$ is shown in Fig. 3 along with AE8-MAX (with internal magnetic field model: Jensen and Cain 1960) predictions. It can be seen that the AE8 peak flux is located more southward than the peak of the CPD fluxes. Furthermore, the AE8 model predicts higher electron fluxes in the auroral ovals than in the SAA. Also the latitude extent of the AE8 trapped electrons reaching the SAA region is reduced as compared to the CPD observations.

No geomagnetic disturbance was observed around 11 April 1999 at 14:52 UT and no flux enhancement was observed in electron fluxes $(1.5<\mathrm{E}<14 \mathrm{MeV})$ measured during this period by the LEO satellite SAMPEX. Therefore, the observed flux of energetic electrons is close to the actual av- eraged values. This is also supported by the higher fluxes obtained on Oersted orbit using the AE-8MIN Update ESASEE1 model available in SPENVIS as compared to AE-8 MAX.

However, before any use of the CPD electron fluxes for modelling purposes, further analysis of the variation of electron fluxes with geomagnetic disturbances, along with investigations of the angular distribution effects should be performed to reach definitive conclusions.

Energetic proton fluxes measured by the CPD are shown in Fig. 4.

The proton fluxes measured by the CPD cover the $1.2<\mathrm{L}<1.8$ range but direct measurements of the equatorial fluxes have been only carried out around $\mathrm{L} \sim 1.2$. The flux at magnetic equator for higher values of the Mcllwain parameters may be derived whenever the actual local pitch angle distribution is known (Fischer et al., 1977; Daly et al., 1996). However, this extrapolation procedure is only valid if the parameter $n$ of the pitch angle distribution is constant, i.e. when particles are not lost by interaction with the upper atmosphere. In any case, the systematic error due to the isotropy assumption on the measured fluxes must be evaluated before further uses of the derived fluxes. Since more important differences have been observed between proton fluxes measured by several instruments than between electron fluxes from different data sources, the evaluation of the anisotropy effect on measurements is performed for protons only.

\section{Effect of the proton flux anisotropy on CPD measure- ments in the SAA}

The zero order approximation of the proton flux, $\mathrm{J}_{C P D}$, is the averaged value over the detector field of view. This isotropic flux approximation is derived from the counting rates using the method of intrinsic detection efficiencies. As already stated, resorting to efficiencies allows discrimination of particle types contributing to the counting rates.

Afterwards, the measured isotropic flux $\mathrm{J}_{C P D}$ is used to derive the omni-directional flux that may be used for applications or model validation purposes. If the pitch angle distribution is assumed to be described by the two parameter model $J(\alpha)=J_{\perp} \sin ^{n} \alpha\left(J_{\perp}\right.$, is the unidirectional flux in the direction perpendicular to the magnetic field vector and $n$ is a parameter related to the steepness of the pitch angle distribution) and if the azimuthal distribution has an axial symmetry around the magnetic field vector, then the averaged flux measured by the CPD may be expressed as

$$
J_{C P D}=J_{\perp} \int_{\beta-\Delta \theta}^{\beta+\Delta \theta} \sin ^{n+1} \alpha d \alpha / \int_{\beta-\Delta \theta}^{\beta+\Delta \theta} \sin \alpha d \alpha
$$

where $\beta$ is the detector orientation angle, i.e. the angle between the detector axis and the local magnetic field vector; and $\Delta \theta$ is the detector FOV half angle. 
On the other hand, the omni-directional flux may be expressed as

$$
\frac{J_{0}}{4 \pi}=J_{\perp} \int_{0}^{\pi / 2} \sin ^{n+1} \alpha d \alpha
$$

Combining equation (1) and (2), one gets

$$
\begin{aligned}
\frac{J_{0}}{4 \pi} & =J_{C P D} \frac{\int_{0}^{\pi / 2} \sin ^{n+1} \alpha d \alpha}{\int_{\beta-\Delta \theta}^{\beta+\Delta \theta} \sin ^{n+1} \alpha d \alpha} \int_{\beta-\Delta \theta}^{\beta+\Delta \theta} \sin \alpha d \alpha \\
& =J_{C P D} g(\beta, n)
\end{aligned}
$$

The $\mathrm{g}(\beta, n)$ function relates the omni-directional flux $J_{0}$ to the measurements obtained from the CPD. It is well known that the pitch angle distribution has the form $\mathrm{F}(\alpha) \sim \sin ^{n}(\alpha)$ only in space position where the Liouville's theorem applies. At those positions $n$ is constant over the whole magnetic field line and the motion of the particle is determined by the magnetic field. When the pitch angle is near the loss cone a particle interacts with atoms in the upper atmosphere in addition to the magnetic field, and the pitch angle distribution fall off more steeply than does the $\sin ^{n}(\alpha)$ law. This feature was observed within the Oersted/CPD data set. To analyse the effect of the departure from the $\sin ^{n}(\alpha)$ law function on the relationship between $\mathrm{J}_{0}$ and $\mathrm{J}_{C P D}$, the pitch angle distribution was linearly extrapolated from the inflection point angle $\alpha_{c}=\arctan (\sqrt{n-1})$ or $\pi-\alpha_{c}$ down to the loss cone angle. This function represents a proxy for the worst case where a detector can be abruptly oriented into the loss cone.

The Fig. 5 shows the shape of the $\mathrm{g}(\beta, n)$ function for a detector having a $67 \mathrm{deg}$. F.O.V angle in case of a $\sin ^{n}(\alpha)$ pitch angle distribution, a $67 \mathrm{deg}$. F.O.V. angle in case of a steep pitch angle distribution and a detector with a $90 \mathrm{deg}$. F.O.V angles and a $\sin ^{n}(\alpha)$ pitch angle distribution, respectively. There is not significant difference between the $\mathrm{g}(\beta, n)$ functions calculated for the two pitch angle distributions. The actual Oersted/CP measurement conditions are between the case shown in Fig. 5a and that of Fig. 5b. For detector orientations around 60 deg., Figs. $5 \mathrm{a}$ and $\mathrm{b}$ show that $\mathrm{g}(\beta, n) \sim 0.7$. Figs. 5a and c may be used to compare detectors having different F.O.V. angles. Of course this kind of analysis for the $\mathrm{g}$ function is required for any other implied angular distribution. However, whenever the number of detector channels allows it, a global fit of the energy and angle distribution should be performed.

For a particle telescope having a given field of view half angle $\Delta \theta$, this function varies slightly with the pitch angle distribution parameter $n$ and rather steeply as a function of the orientation angle out of the range $\pi / 2-\Delta \theta \leq \beta \leq \pi / 2+\Delta \theta$. For the CPD, in this orientation angle interval, values of $\mathrm{g}(\beta, n)$ range from 0.55 and 1 for $n$ values between 0 and 50 . Therefore, measurements performed by the CPD in space regions characterized by the orientation and pitch angle distribution parameter in the above given range may be considered to be equivalent to the omnidirectional flux divided by $4 \pi$, within a factor less than two.
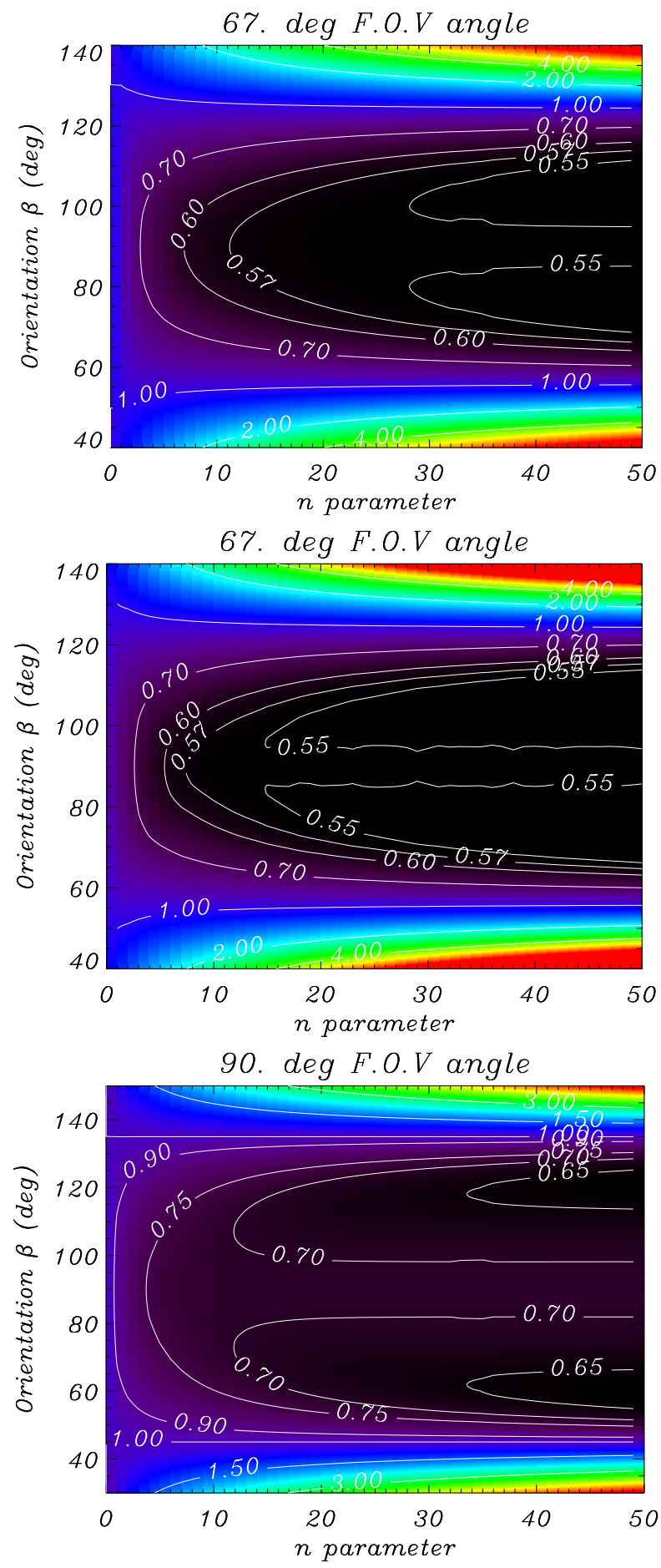

Fig. 5. Function $\mathrm{g}(\beta, n)$ for: (a) a $\Delta \theta \mathrm{g} 33.5 \mathrm{deg}$. F.O.V. half-angle detector like the CPD with a $\sin ^{n}(\alpha)$ pitch angle distribution; (b) a $\Delta \theta \mathrm{g} 33.5 \mathrm{deg}$. F.O.V. half-angle detector with a steep pitch angle distribution; and (c) a $\Delta \theta=45$ deg. F.O.V. half-angle detector.

It must be noticed that even if the CPD was oriented perpendicularly to the magnetic field it would not be suspected of measuring extremely overestimated values of fluxes: such fluxes would be only by a factor of 2 higher than values 


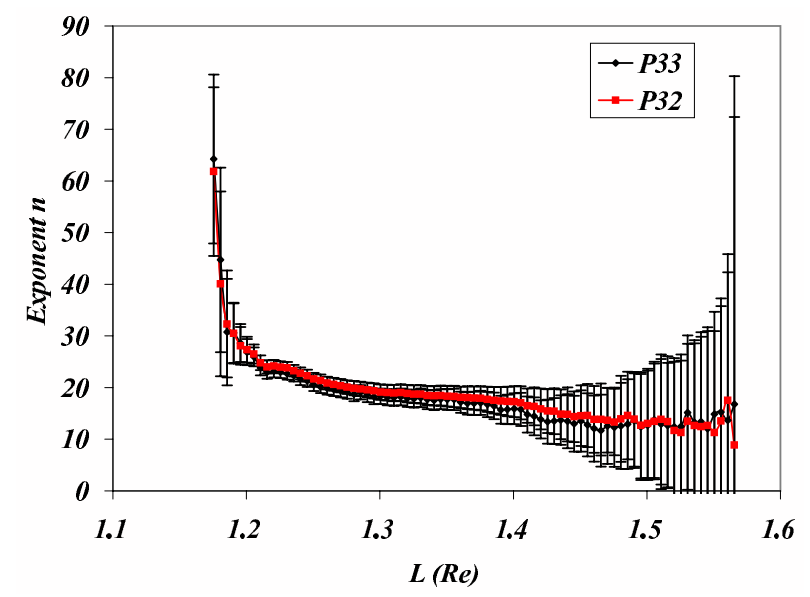

Fig. 6. Pitch angle distribution parameter $n$ as a function of the McIlwain L coordinate for the $20-80 \mathrm{MeV}$ (black) and $40-100 \mathrm{MeV}$ (red) energy channels. High values of statistical uncertainty at the lower and upper ends of the $L$ range result from poor statistics.

obtained by dividing omni-directional fluxes by $4 \pi$.

In general, from a model of pitch angle distribution in space positions and a model of the magnetic field, the $\mathrm{g}(\beta, n)$ function can be evaluated for any telescope and included into the raw data to be used in omni-directional flux evaluations. Models of proton pitch angle distribution at low altitude have been developed since the discovery of the radiation belt. However, although a huge amount of work has been accomplished towards the implementation of angular distribution models into the Space Environment Information System (SPENVIS), none of them was made available for public access yet and they probably need further validation (Lemaire et al., 1998; Heynderickx et al., 2004). We resorted to the method described in (Fischer et al., 1977) to determine the parameter $n$ as a function of the McIlwain L drift shell coordinates. The results are shown in Fig. 6.

Using these values of the parameter $n$ characterising the positions sampled by the Oersted/CPD and the orientation angle distributions in the SAA, calculated using IGRF 1995, shown in Fig. 7, it may be concluded that an average value of the $\mathrm{g}(\beta, n)$ function equal to 0.7 may be applied to roughly evaluate the omni-directional flux.

The function $\mathrm{g}(\beta, n)$ was obtained under the assumption of an axial symmetry of the azimuthal angle distribution. The East-West asymmetry was neglected since the involved angular distribution is much less steep than the pitch angle distribution. However, in a thorough evaluation of omnidirectional fluxes based on measurements performed using instruments like the CPD, this East-West asymmetry should be taken into account.

All in all, full angular distribution (including pitch angle and azimuthal angle) should be considered as an important part of models of particle fluxes at low altitude. Charged particle telescope should comprise capability to measure angular distributions to be used in the development of accurate flux model for low altitudes.
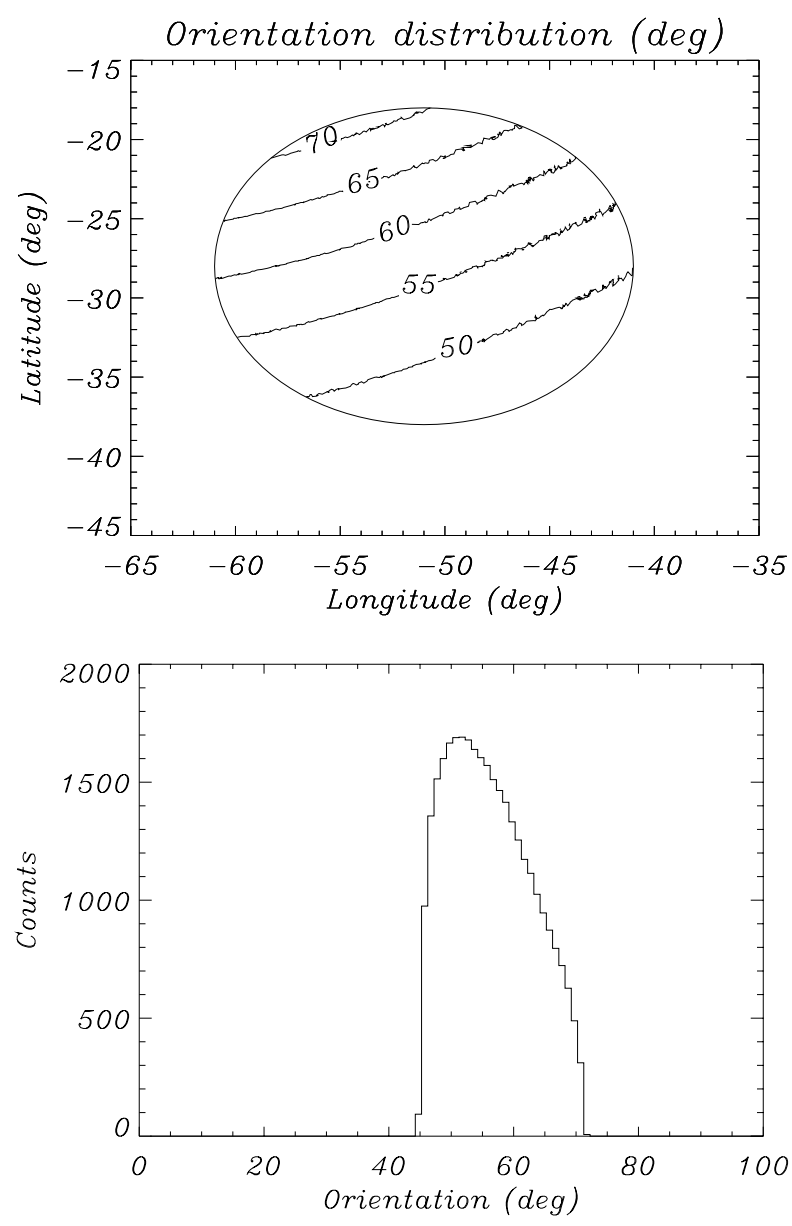

Fig. 7. (a) Geographical positions within a $10^{\circ}$ range around the position of peak flux defined herein as the heart of SAA throughout this paper and contour of constant orientation angle $\beta$. (b) Histogram of orientation angles $\beta$ sampled by the Oersted/CPD within the SAA region.

\section{Comparison with AP8-MAX, SAMPEX/PET and PROBA/SREM results}

Comparisons of Oersted/CPD data to other proton fluxes are based on Eq. 3. AP8 omni-directional fluxes $\mathrm{J}_{0}$ are divided by $4 \pi$ and compared to unidirectional fluxes measured by the Oersted/CPD or to modelled unidirectional fluxes accessed by selecting the SAMPEX/PSB97 model in SPENVIS. The function $\mathrm{g}(\beta, n)$ is taken into account whenever possible, otherwise estimates of its average over the data set may be used. When directly compared to AP8-MAX and SAMPEX/PSB97 predictions, the flux of energetic protons $\mathrm{J}_{C P D}$ is more than an order of magnitude higher than AP8-MAX and at most a factor 3 higher than SAMPEX/PSB97 predictions. This factor is reduced to $\sim 2$ if an average value of $\mathrm{g}(\beta, n)$ equal to 0.7 is considered. For Oersted/CPD, the influence of energetic particles that may come from the back side of the satellite was assessed (Cyamukungu et al., 2001b). It was concluded that their contribution to the total counting rates could not induce this order of magnitude difference. A 
variant of the background analysis was presented in Sect. 2 .

The fluxes from PROBA1/SREM are in good agreement with AP8-MAX within a factor of two, whereas predictions from the TPM-1 proton flux model derived from instruments on board the NOAA satellites TIROS fall between the corrected Oersted/CPD fluxes and SAMPEX/PSB97 predictions (Buehler, 2003; Xapsos 2002). AP8-MAX, SAMPEX and $\mathrm{J}_{C P D}$ results are shown in Fig. 8 as energetic proton fluxes versus the altitude.

We have shown that detectors are inaccurate for low altitude flux measurements on non spinning satellites if they have small FOV angle and are oriented toward the $90^{\circ}$ pitch angle or whenever they are oriented towards the loss cone. Detectors having a wide FOV angle appear to measure the most accurately the omni-directional fluxes for various shapes of pitch angle distributions and for a wide range of orientation angle. Unfortunately, data set with energetic particle fluxes measured by accurate detectors in low altitude orbit are scarcely available. The AP8 model is used at low altitude mainly because it is generally admitted that it underestimates the energetic proton fluxes by a factor $\sim 2$ only. Further uses of this model for low altitude flux predictions lay on the non-confirmation of the Oersted/CPD, SAMPEX/PET and NOAA/TIROS results. Therefore, instruments and missions to measure full angular distributions (pitch and azimuth angle) and fluxes of energetic particles are of great interest.

\section{Conclusions}

The main features of the energetic electron fluxes measured by the CPD on board the Oersted satellite have been outlined. The peak value of the electron flux is in good agreement with AE8-MAX predictions, whereas the position of this peak intensity and the spatial extension are different.

The flux of energetic protons is significantly higher than AP8-MAX predictions. It has been demonstrated that the order of magnitude difference between AP8-MAX and Oersted/CPD results does not come from any systematic effect that might have resulted from the orientation of the CPD towards the maximum of the pitch angle distribution. Available data sets containing particle fluxes at low altitude should be validated by checking whether most of the data were not acquired while the instruments were looking into the loss cone or not. As a matter of fact the development of publicly available angular distribution models for particles at low altitudes should be encouraged along with the development of background-free instruments to acquire the data needed for the study of angular distribution dynamics.

Acknowledgements. The authors are grateful to D. Boscher, P. Buehler and D. Heynderickx for information they have provided regarding the observations made using several instruments in the SAA.

A. Leonov thanks INTAS for the support given to him through the Young Scientist Fellowship at the Center for Space Radiation in Louvain-la-Neuve.

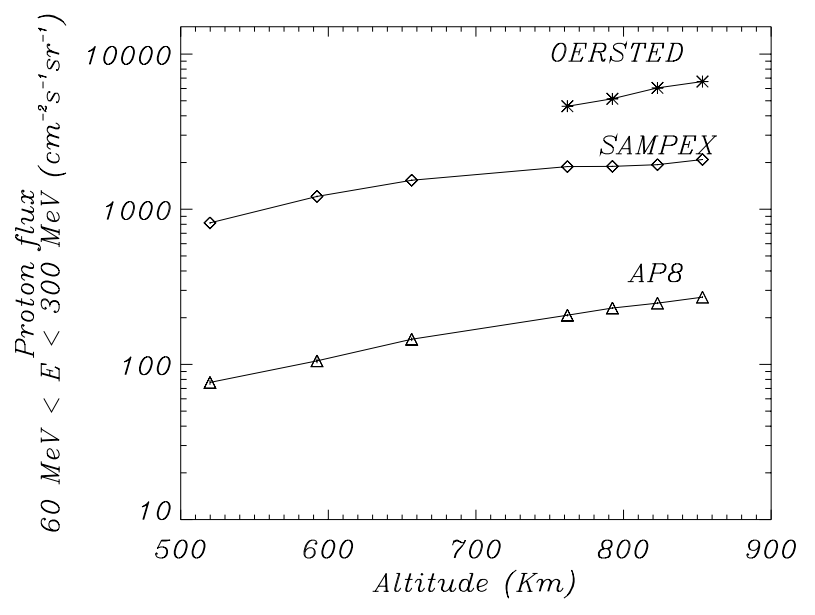

Fig. 8. Flux of energetic protons as a function of altitude from Oersted/CPD (uncorrected, from April 1999 data) measurements compared to SAMPEX/PSB97 and AP8-MAX predictions. The typical statistical error on proton fluxes are $10 \%$ and the systematic error was considered negligible.

The Oersted/CPD instrument operations are supported by the Danish Meteorological Institute and data analysis tasks were supported by the PRODEX-ESA-SSTC Contract No. 170724 and partly funded by ESA through the Radiation Environment Research with Multiple Monitor (RERMM) lead by S. Bourdarie at ONERA, France. We wish also to thanks J. B. Blake for his constructive remarks. The authors express thanks to COST 724 for its contribution in this publication.

Topical Editor T. Pulkkinen thanks G. Esposito and E. Fiandrini for their help in evaluating this paper.

\section{References}

Agostinelli, S., Allison, J., Amako, K., et al.: Geant4 - a simulation toolkit; Nuclear Instruments and Methods in Physics Research, A506, 250-303, 2003.

Bidoli, V., Canestro, A., Casolino, M., et al.: In-orbit performance of the space telescope NINA and galactic cosmic-ray flux measurements; Astrophy. J. S., 132, 365-375, 2001.

Buehler, P.: Technical Note: PROBA-1/SREM Data Access and Instrument Information WP110 - Radiation Environment Research from Multiple Monitors, ESTEC Contract No. 16709/02/NL/EC; Issue 1.0, 2003.

Cyamukungu, M., Grégoire, G., Heynderickx, D., Kruglanski, M., Lemaire, J., Blake, J. B., and Selesnick, R. S.: Proton Spectra Detected by the Proton Switches on the CRRES Satellite; J. Spac. Rock., 38, 4, 584-589, 2001a.

Cyamukungu, M., Stauning, P., Grégoire, G., and Lemaire, J.: The Charged Particle Detector (CPD): Electron and Proton spectra - Technical Note B - PRODEX-ESA-SSTC, ContractNo. 170724, also available at: http://www.spaceradiations.be/en/ activities/index.html, 2001b .

Daly, E. J., Lemaire, J., Heynderickx, D., and Rodgers, D. J.: Problems with Models of the Radiation Belts; IEEE Trans. Nucl. Sci., 43, 2, 403-415, 1996.

Fischer, H. M., Auschrat, V. W., and Wibberenz, G.: Angular Distribution and Energy Spectra of Protons of Energy $5 \leq E \leq 50 \mathrm{MeV}$ 
at the Lower Edge of the Radiation Belt in Equatorial Latitudes; J. Geophys. Res., 82, 4, 537-547, 1977.

Heynderickx, D., Quaghebeur, B., Wera, J., Daly, E. J., and Evans, H. D. R.: New radiation environment and effects models in ESA's SPace ENVironment Information System (SPENVIS), Space Weather, 2, doi:10.1029/2004SW000073, also available at: http://www.spenvis.oma.be/spenvis/, 2004.

Lemaire, J., Heynderickx, D., Kruglanski, M., Johnstone, A. D., Rodgers, D. J., Szita, S., Jones, J., Keppler, E., Friedel, R., and Loidl, G.: TREND-3 Radiation Environments of Astronomy Missions and LEO Missions - Final Report ESTEC Contracts No. 10725/94/NL/JG(SC) and No. 11711/95/NL/JG(SC) WO-3, 35-78, 1998.
Leonov, A., Cyamukungu, M., Cabrera, J., Leleux, P., Lemaire, J., Gregorie, Gh., Mikhailov, V., Bakaldin, A., Galper, A., Koldashov, S., Voronov, S., Casolino, M., De Pascale, M., Picozza, P., Sparvolli, R., Ricci, M.: Pitch angle distribution of trapped energetic protons and helium isotopes nuclei measured along the Resurs-01 No.4 LEO satellite, Ann. Geophys., 23, 2983-2987, 2005.

Xapsos, M. A., Huston, S. L., Barth, J. L., Stassinopoulos, E. G.: Probabilistic Model for Low-Altitude Trapped-Proton Fluxes; IEEE Trans. Nucl. Sci., 49, 6, 2776-2781, 2002. 